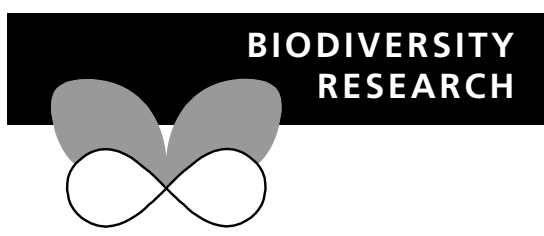

\title{
The role of landscape texture in conservation biogeography: a case study on birds in south-eastern Australia
}

\author{
Joern Fischer, David B. Lindenmayer and Rebecca Montague-Drake
}

The Fenner School of Environment and Society,

The Australian National University, Canberra, ACT 0200, Australia

${ }^{*}$ Correspondence: Joern Fischer, The Fenner School of Environment and Society, The Australian National University, Canberra, ACT 0200, Australia. Tel.: +61 261254612; Fax: +61 261250757;

E-mail: joern.fischer@anu.edu.au

\begin{abstract}
The binary classification of landscapes into suitable vs. unsuitable areas underlies several prominent theories in conservation biogeography. However, a binary classification is not always appropriate. The textural discontinuity hypothesis provides an alternative theoretical framework to examine the geographical distribution of species, and does not rely on a binary classification scheme. The texture of a given landscape is the combination of its vertical structural complexity and horizontal spatial grain. The textural discontinuity hypothesis states that biophysical features in the environment are scaled in a discontinuous way, and that discontinuities in the body size distribution of animals mirror these biophysical discontinuities. As a result of this relationship, a complex landscape texture should be associated with small-bodied animals, whereas a simple landscape texture should be associated with larger-bodied animals. We examined this hypothesis for birds in five landscapes in south-eastern Australia that represented a gradient from simple to complex landscape texture. In landscapes with a complex texture, the number of detections of small birds was higher than expected, and the number of detections of larger-bodied birds was lower than expected. The opposite pattern was found in landscapes with a simple texture. The pattern remained significant when only bird species found in each of the five landscapes were considered, which demonstrated that the association of landscape texture with body size was not an artefact of landscapes differing in their species pools. Understanding the effects of landscape texture on species distribution patterns may be a promising research frontier for conservation biogeography. We hypothesize that the active management of landscape texture may be used to attract or deter animals of certain body sizes. Consistent with other theories, the textural discontinuity hypothesis therefore suggests that managing entire landscapes, rather than only predefined patches, is an important conservation strategy.
\end{abstract}

\section{Keywords}

Birds, body mass distribution, landscape heterogeneity, textural discontinuity hypothesis, vegetation structure.

\section{INTRODUCTION}

The emerging field of conservation biogeography applies 'theories [on] the distributional dynamics of taxa ... to problems concerning the conservation of biodiversity' (Whittaker et al., 2005). To date, much of the focus of conservation biogeography has been on island biogeography (MacArthur \& Wilson, 1967; Watson et al., 2005), nested subset theory (Patterson, 1987; Hylander et al., 2005), and reserve selection (Diamond, 1975a; Meir et al., 2004). Implicit to these themes is the binary classification of land into ecologically suitable vs. unsuitable conditions (Haila, 2002). In their special paper on conservation biogeography, Whittaker et al. (2005) acknowledged that this binary classification can be overly simplistic because conditions in the matrix surrounding predefined patches may affect the distribution of taxa (Watson et al., 2005). Indeed, evidence that 'the matrix matters' (Ricketts, 2001) is now overwhelming (Daily, 2001; Lindenmayer \& Franklin, 2002; Manning et al., 2004; Kupfer et al., 2006), and conservation biogeography urgently needs tools to formally account for the effects that entire landscapes (rather than patches alone) have on the distribution of species. Although a range of concepts may be useful to expand the focus from patches to entire landscapes (e.g. Tscharntke et al., 2005; Bennett et al., 2006), in this paper, we are concerned with a single specific hypothesis whose potential contribution to conservation biogeography has not been recognized to date. 
We propose that the 'textural discontinuity hypothesis' (sensu Holling, 1992) offers a promising theoretical background against which to examine and manage the distribution of species at the landscape to regional scale. First, we outline the textural discontinuity hypothesis in the context of a hierarchical framework that attempts to explain why large or small species occur in a given area. Second, using empirical data, we demonstrate the effects of landscape texture on the distribution of birds of different body sizes in five landscapes in south-eastern Australia. We discuss our findings in the context of general ecological theory, and we outline possible conservation implications of the textural discontinuity hypothesis.

\section{THEORETICAL FOUNDATION AND PREDICTIONS}

The frequency distribution of body mass in animals is typically right-skewed and can exhibit marked discontinuities (Holling, 1992; Hubbell, 1997; Gardezi \& da Silva, 1999; Allen, 2006). Allen et al. (2006) recently reviewed several potential hypotheses for this pattern and suggested that different explanations are likely to be satisfactory at different spatial and temporal scales.

At the continental scale, body mass distributions may be influenced by energetic constraints dictating certain optimal body sizes (Brown et al., 1993). Phylogenetic relationships and biogeographical processes also may influence body mass distributions at large scales (Polo \& Carrascal, 1999; Siemann \& Brown, 1999; Allen et al., 2006).

At the landscape scale, the structure and spatial grain of vegetation cover may favour certain body sizes over others (Gunnarsson, 1992; Telleria \& Carrascal, 1994; McAbendroth et al., 2005). The 'textural discontinuity hypothesis' (Holling, 1992) states that biophysical features in the environment are scaled in a discontinuous way, and that discontinuities in the body size distribution of animals mirror these biophysical discontinuities. Holling (1992) distinguished between landscapes with a complex texture (high structural complexity and fine-grained heterogeneity) and landscapes with a simple texture (low structural complexity and coarse-grained heterogeneity). According to the textural discontinuity hypothesis, smallerbodied animals are associated with landscapes with a complex texture, whereas larger-bodied animals are associated with landscapes with a simple texture (Holling, 1992; Allen, 2006). Within a given landscape, interspecific processes such as competition may result in animals of different body sizes being able to coexist at a given site because they exploit different resources (Hutchinson \& MacArthur, 1959).

We investigated differences in the body mass distribution of birds occupying five different landscapes in south-eastern Australia. The landscapes represent a continuum of structure and spatial grain of vegetation cover, and have in common some but not all species of birds. Similar survey protocols were used in all landscapes to document the presence and number of detections of birds. The resulting data set lends itself well to an analysis of the relationship between landscape texture and the body mass distribution of birds.
Following Holling (1992) and Allen et al. (2006), we posed two specific hypotheses:

1 The number of detections of birds in different body size classes should differ systematically between landscapes. The number of detections of small birds should be higher than expected in landscapes with a complex texture, and lower than expected in landscapes with a simple texture. Conversely, the number of detections of large birds should be lower than expected in landscapes with a complex texture, and higher than expected in landscapes with a simple texture.

2 The abundance patterns of those species present in all landscapes should vary systematically with landscape texture. A given small species should be detected most frequently in structurally complex landscapes, whereas a given large species should be detected most frequently in structurally simple landscapes.

There has been considerable controversy over whether body mass has a continuous or discontinuous distribution (Holling, 1992; Siemann \& Brown, 1999). We are not concerned with this debate in this paper, although we acknowledge that the notion of discontinuities was central to Holling's (1992) original proposition of the textural discontinuity hypothesis. In contrast, our objective is to assess the relationship between landscape texture and body mass, irrespective of whether the range of body masses among different species has a continuous or discontinuous distribution.

\section{METHODS}

\section{Study sites and data collection}

We examined five landscapes in south-eastern Australia. In all landscapes, similar point count protocols were used by multiple experienced observers. This enabled the valid comparison of results across data sets. In all cases, a given point count lasted $5 \mathrm{~min}$.

The landscape with the most complex texture was the Central Highlands landscape in Victoria $\left(-37.5^{\circ}, 145.6^{\circ}\right.$; Fig. 1$)$ (Lindenmayer et al., 1999). This landscape was dominated by tall, wet eucalypt forest. The overstorey was dominated by Mountain Ash (Eucalyptus regnans) or Alpine Ash (Eucalyptus delegatensis), and the understorey was typically dense, containing several species of Acacia as well as tree ferns (Dicksonia antarctica and Cyathia australis) and a range of shrub species. Bird data were collected at 87 sites in spring 2004 and spring 2005. At the vast majority of sites, two observers conducted point counts at three locations spaced $50 \mathrm{~m}$ apart, and birds seen or heard within $50 \mathrm{~m}$ were recorded (total number of point counts = 1029). Cunningham et al. (1999) showed that pooling data gathered by two or more observers effectively accounted for between-observer heterogeneity in their ability to detect certain groups of birds.

Two adjacent landscapes with intermediate landscape texture were located in the Tumut region of New South Wales $\left(-35.2^{\circ}\right.$, 148.6º Fig. 1) (Lindenmayer et al., 2002). The 'Tumut continuous forest' landscape was relatively unmodified eucalypt forest 

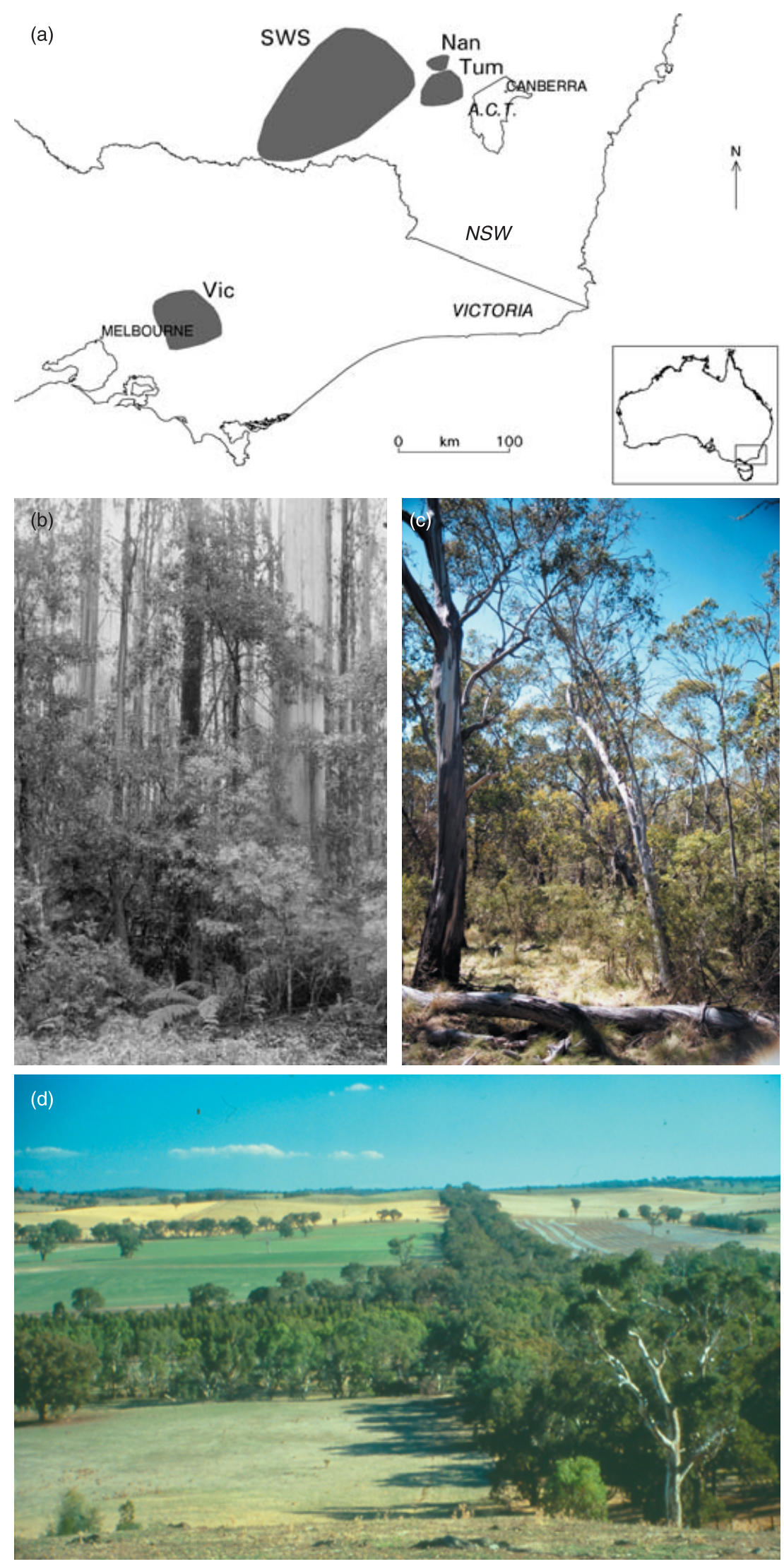

Figure 1 Location and photographs of the landscapes examined in this paper. (a) The map shows the locations of Nanangroe (Nan), Tumut (Tum), the Central Highlands of Victoria (Vic), and the south-west slopes (SWS). (b) Landscape texture was fine-grained and complex in Victoria, intermediate in Tumut (c), and coarse-grained and simplified in Nanangroe and the south-west slopes (d). 
dominated by narrow-leaved peppermint (Eucalyptus radiata), ribbon gum (Eucalyptus viminalis), and to a lesser extent red stringybark (Eucalyptus macrorhyncha) and swamp gum (Eucalyptus camphora). The understorey in this landscape contained a range of Acacia spp. as well as native shrubs, but compared to the Central Highlands landscape, vegetation structure was more open. Bird data were collected at 40 sites in spring 1997 and spring 1998. Sites were visited by at least two different experienced observers. Seven point counts were conducted at any given site, spaced at $100 \mathrm{~m}$ intervals (total number of point counts $=584$ ). During point counts, all birds heard or seen within the site were recorded.

The 'Tumut remnant forest' landscape was a plantation of the introduced radiata pine (Pinus radiata) throughout which remnant patches of eucalypt forest had been retained. The remnant patches varied in size from $<1$ ha to $>100$ ha. They were broadly similar in structure and composition to the nearby continuous forest landscape, with the exception of the widespread presence of introduced blackberry thickets (Rubus fruticosus). Bird data were collected in 84 remnant patches in spring 1996 and in 85 patches in spring 1997. The survey protocol was the same as in continuous forest (total number of point counts $=1289$ ).

Two additional landscapes with a simpler landscape texture were examined. The Nanangroe landscape $\left(-35.0^{\circ}, 148.5^{\circ}\right.$; Fig. 1) was located approximately $40 \mathrm{~km}$ to the north-west of the Tumut region and was used for sheep (Ovis aries) and cattle (Bos taurus) grazing (Lindenmayer et al., 2001). Prior to European settlement, this landscape was dominated by open, grassy woodland vegetation, but approximately $85 \%$ of native vegetation has been cleared since then. Remnant woodland patches varied in size from $<1$ ha to approximately 15 ha. The overstorey was dominated by yellow box (Eucalyptus melliodora), white box (Eucalyptus albens), Blakely's red gum (Eucalyptus blakelyi), and to a lesser degree red stringybark. Compared to the Central Highlands landscape and the Tumut landscapes, overstorey trees were spaced widely, with adjacent crowns of trees frequently not touching. The understorey was simple, which is partly natural for grassy woodlands and partly the result of persistent grazing pressure from domestic livestock (Prober \& Thiele, 1995). Shrubs and tall native grasses were uncommon throughout the Nanangroe landscape. Bird data were collected at 55 woodland sites in both spring 2003 and spring 2005. The vast majority of sites contained three point count stations at $100-\mathrm{m}$ intervals. Sites were visited by at least two different observers, and birds seen or heard within $50 \mathrm{~m}$ during a given point count were recorded (total number of point counts $=662$ ).

Finally, we examined the south-west slopes landscape in New South Wales $\left(-35.7^{\circ}, 147.3^{\circ}\right.$; Fig. 1$)$, which was located approximately $100 \mathrm{~km}$ to the west of Nanangroe. The land-use history of this landscape was broadly similar to that of the Nanangroe landscape, but land use included cropping as well as domestic livestock grazing. Remnant vegetation in the area was broadly similar in structure and species composition to Nanangroe, with the exception that some tree species (e.g. white box) were generally less abundant than at Nanangroe while others were typically more abundant (e.g. red stringybark). In spring 2002 and spring 2004, bird data were collected in 129 and 139 woodland patches, respectively, varying in size from $<1$ ha to 40 ha. The survey protocol was the same as at Nanangroe (total number of point counts $=1623$ ).

In summary, our study investigated bird communities in five landscapes representing three broad levels of landscape texture: complex, fine-grained texture in the Central Highlands, intermediate texture in continuous forest and forest remnants at Tumut, and simple, coarse-grained texture at Nanangroe and in the south-west slopes (Fig. 1).

\section{Data analysis}

First, we tested whether the overall distribution of body masses differed between landscapes. We compiled species lists for each landscape and compared the mean body mass of birds and its associated variability between landscapes using analysis of variance. Body mass values for each species were compiled from the published literature, mostly from the Handbook of Australian, New Zealand \& Antarctic Birds (Davies et al., 1991; Marchant et al., 1994; Higgins \& Davies, 1996; Higgins, 1999; Higgins et al., 2001; Higgins \& Peter, 2003; Higgins et al., 2006; see Table S1 in Supplementary material). Although this first step in our analysis was not a direct test of the textural discontinuity hypothesis, it was useful to test for potential differences in body mass distribution at a general level.

Second, we tested whether the number of detections of birds in different body mass groups differed systematically between landscapes (hypothesis 1). We used hierarchical clustering analysis with the complete linkage method in $R$ to assign each species recorded in any of the surveys to one of 10 body mass groups. We chose this method because it is useful and intuitive to detect natural breaks in the data. Body mass was log-transformed prior to this analysis (see Allen et al., 1999). Because only a single species occurred in the group of the largest birds, we pooled the two groups containing the largest birds prior to further analyses.

For each species, we recorded the number of point count stations in each landscape where it was recorded. We then summed the number of detections across all species within each body mass group. This 'frequency count' was cross-tabulated for each of the five landscapes (columns) by the nine body mass groups (rows). We conducted a chi-squared test of homogeneity across the columns and plotted the Pearson residuals from this analysis to visualize whether frequency counts in each body mass group differed systematically between landscapes.

Third, we tested whether the number of detections of those species recorded in all five landscapes differed systematically with their body size (hypothesis 2). We calculated the proportion of detections per unit of survey effort for each of the ubiquitous species in each landscape (e.g. for species X, per unit of survey effort, $20 \%$ of detections came from the Central Highlands, 30\% from Nanangroe, and so on). We sorted species by the ratio of detections in the landscapes with simple texture (Nanangroe and south-west slopes) over detections in the three other landscapes with more complex texture. Each species was assigned a 'row 


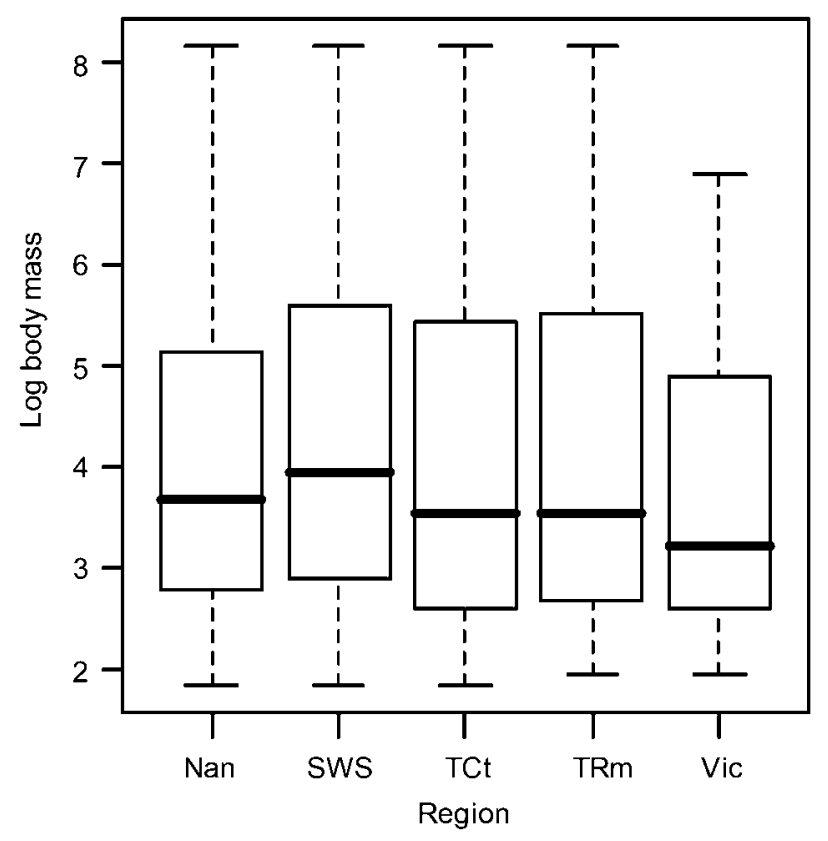

Figure 2 The overall distribution of the body mass of birds (in grams, log-transformed) did not differ significantly between landscapes $(P=0.49$; Nan, Nanangroe; SWS, south-west slopes; TCt, Tumut continuous forest; TRm, Tumut remnant forest; Vic, Central Highlands forest).

position' on the basis of this sorting algorithm. We tested whether a species' row position was significantly related to its log body mass using simple linear regression.

\section{RESULTS}

Across all landscapes, 161 bird species were recorded (Nanangroe $=101$; south-west slopes $=138$; Tumut continuous forest $=75$; Tumut remnant forest $=83$; Central Highlands $=61$; Table S1).

Our exploratory analysis of the overall distribution of body mass in the different landscapes showed that mean body mass did not differ significantly between landscapes (Fig. 2; $P=0.49$ ).

Our first hypothesis that explicitly examined the textural discontinuity hypothesis could not be rejected. Frequency counts in the nine different body mass groups differed significantly between landscapes $(P<0.0001)$. The nine body mass groups are shown in Fig. 3. A plot of the Pearson residuals showed that departures from expected values were systematic for frequency counts across the five landscapes. The two landscapes with the simplest texture had a lower than expected frequency count of small birds and a higher than expected frequency count of medium-sized birds. In contrast, the three landscapes with more complex texture had a higher than expected frequency count of small birds and a lower than expected frequency count of medium-sized birds (Fig. 4).

The second hypothesis that explicitly examined the textural discontinuity hypothesis also could not be rejected. The number of detections of the 34 species recorded in each of the five landscapes differed systematically with their body size. Species were sorted by the ratio of number of detections in the landscapes with simple texture over the number of detections in landscapes with more complex texture (Fig. 5). Species with a low row position in this sorting algorithm (i.e. more detections in simple landscapes) were significantly larger than species with a high row position (i.e. more detections in complex landscapes; Fig. 6).

\section{DISCUSSION}

When only the presence of birds was considered, there was no significant difference in the body mass distribution of birds across the five landscapes with different texture. This result may be explained in part by all bird species in the five regions originating from the same phylogenetic pool, which may provide a larger-scale constraint on the overall distribution of body masses in the region (Hubbell, 1997; Polo \& Carrascal, 1999; Siemann \& Brown, 1999; Allen et al., 2006). Such a constraint may mean that presence/absence data were too coarse to detect responses of different bird species to landscape texture. In addition, tests of the mean of a statistical distribution may mask the number and location of potential discontinuities in the data. For these reasons, analyses that investigate abundance patterns of birds are more meaningful to assess the textural discontinuity hypothesis.

When the abundance of birds was considered, our results were largely consistent with the textural discontinuity hypothesis (see also Roland \& Taylor, 1997; De la Montana et al., 2006). The number of detections of all 161 species combined deviated systematically from expected values depending on the body size of individual species (Fig. 4). The only exception was that the number of detections of very large birds did not differ from

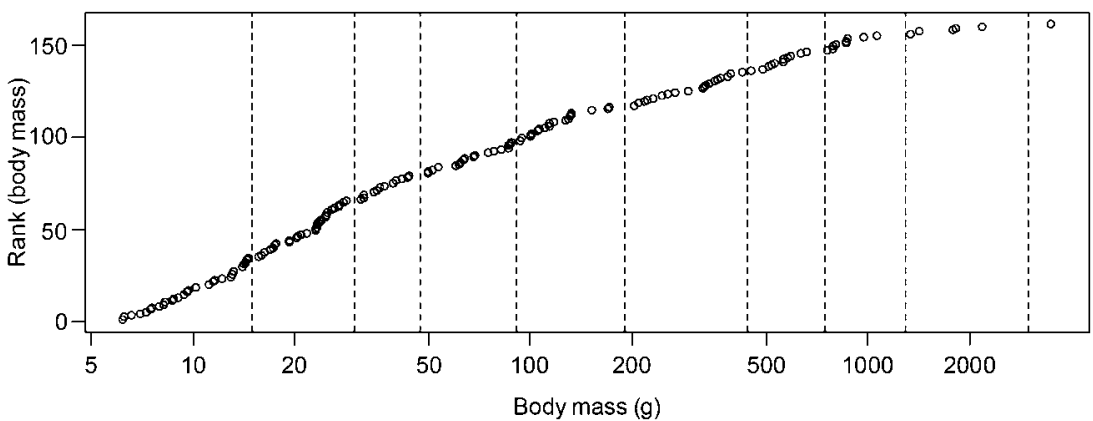

Figure 3 Delineation of body mass groups following hierarchical cluster analysis on the log body mass of the 161 species recorded in any of the five landscapes. The two groups containing the largest birds were pooled for further analyses. 


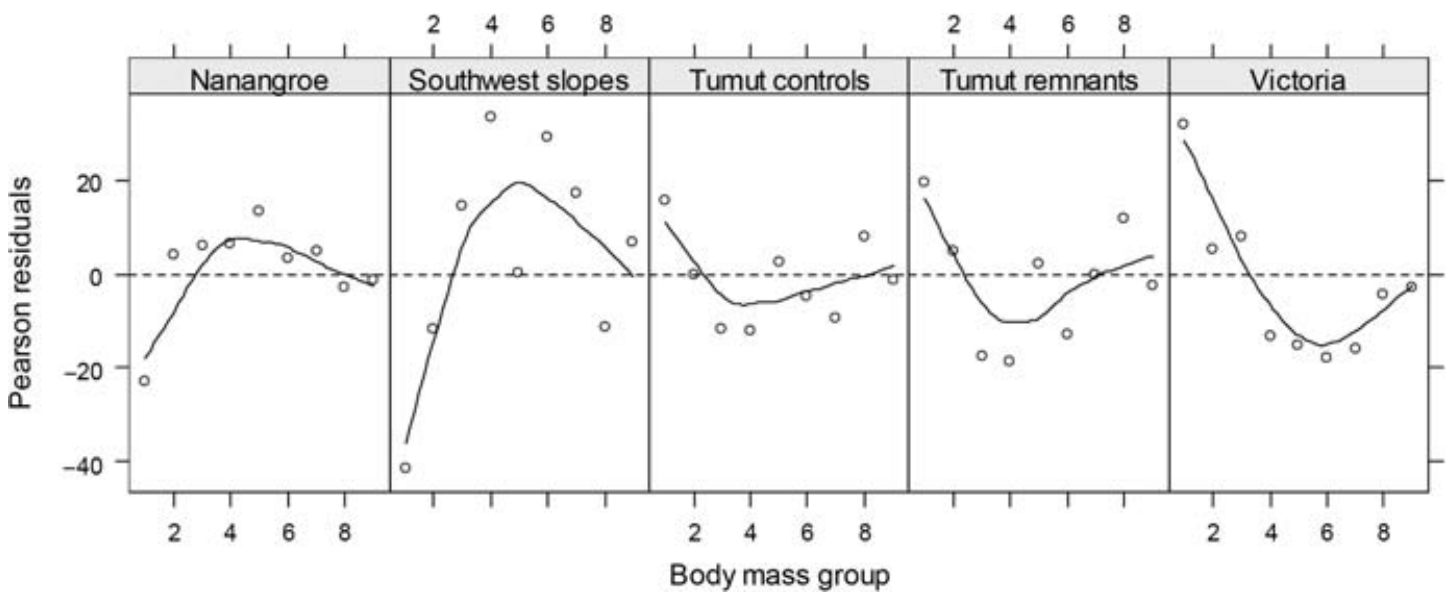

Figure 4 Pearson residuals associated with a chi-squared test of homogeneity for the frequency count of birds across the nine body mass groups. Positive values indicate a higher than expected frequency count and negative values indicate a lower than expected frequency count. Smooth lines were fitted for visualization purposes.

Figure 5 Proportions of detections for each of the 34 species present in each of the five landscapes examined (Vic, Central Highlands forest; TCt, Tumut continuous forest; TRm, Tumut remnants; Nan, Nanangroe; SWS, south-west slopes). Each segment of each species' horizontal bar is the proportion of total detections for a given species in a given landscape out of the five landscapes, having standardized for differences in survey effort. Scientific names of species are listed in Table S1 in Supplementary material.

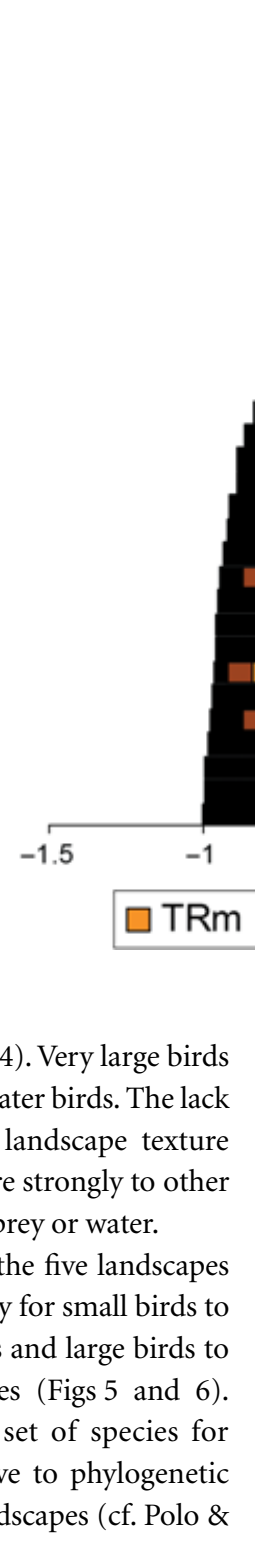

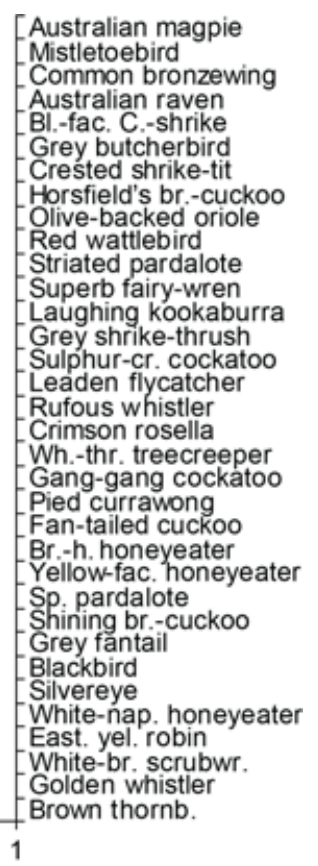

0.5
TCt $\square$ Vic $\square$ Nan $\square$ SWS expected values in any of the landscapes (Fig. 4). Very large birds included several species of birds of prey and water birds. The lack of systematic response by these species to landscape texture most likely indicates that they responded more strongly to other landscape features, such as the availability of prey or water.

When only the species present in each of the five landscapes were analysed, there was a significant tendency for small birds to be more abundant in the complex landscapes and large birds to be more abundant in the simple landscapes (Figs 5 and 6). Because this analysis considered the same set of species for each landscape, the results were not sensitive to phylogenetic differences in the species pools of the five landscapes (cf. Polo \&
Carrascal, 1999). For this reason, this result strongly supports the textural discontinuity hypothesis. As discussed for birds of prey and water birds above, some of the variability in the number of detections for birds of a given body size may be explained by the specific resources used by particular species. The mistletoebird, for example, had a very low row position (Fig. 5), indicating that despite its very small body size $(9.1 \mathrm{~g})$, it was detected most frequently in landscapes with a simple texture. This deviation from the overall pattern most likely relates to the widespread occurrence of mistletoe in the agricultural landscapes with simple texture, which is the primary food source for the mistletoebird. 


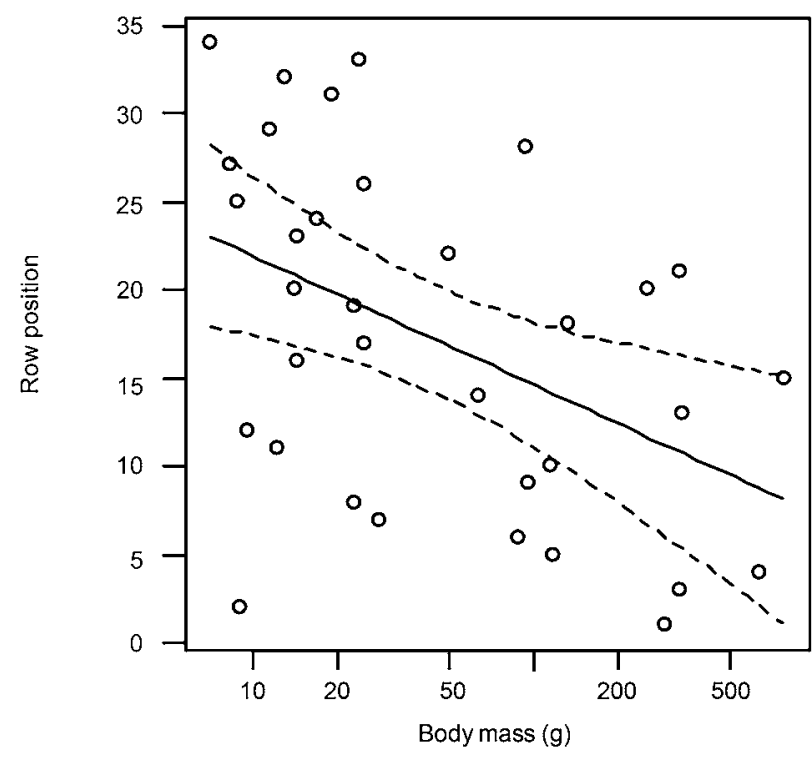

Figure 6 Row position produced by the sorting algorithm in Fig. 5 as a function of each species' log body mass (simple linear regression, $P=0.006$ ). Small birds tended to have a high row position, and large birds tended to have a low row position, indicating that small birds were detected relatively more frequently in landscapes with a complex texture.

Although our results were consistent with the textural discontinuity hypothesis, they only demonstrate a correlation between landscape texture and body mass distribution, but provide no mechanistic explanation. Four mechanistic explanations that are not mutually exclusive are plausible.

First, appropriate shelter opportunities for birds may be related to the interplay between body size and vegetation structure. For example, many small birds shelter in shrubs (Ford, 1985; Grover \& Slater, 1994), whereas many large birds utilize larger branches, and perceive their environment at a coarser spatial grain (Diamond, 1975b; Wiens, 1989). Second, the movement of large birds may be restricted in dense vegetation (Miles \& Ricklefs, 1984), and large distances between habitat features such as scattered trees may be crossed more easily by large bird species (Fischer \& Lindenmayer, 2002). Third, resource partitioning between species allows for many small animals to co-occur at any given site in landscapes with a complex texture (Hutchinson \& MacArthur, 1959; August, 1983; McAbendroth et al., 2005). Fourth, the observed pattern may be an artefact of different landscapes exhibiting different landscape modification states (sensu McIntyre \& Hobbs, 1999). In addition to landscape texture, levels of human impact also differed between the five landscapes we examined. It is possible that small species may have been more extinction-prone in agricultural landscapes, and that they used to be more abundant in these landscapes than they are today. However, it is unlikely that small body size per se caused extinction proneness. The home ranges of smaller species tend to be smaller than those of larger species (Schoener, 1968), and several studies have highlighted that large, rather than small, species are particularly prone to extinction (Renjifo, 1999; Cardillo \& Bromham, 2001; Reynolds, 2003; Cardillo et al., 2005). Hence, if small species were particularly prone to extinction in agricultural landscapes, it is likely that this pattern was caused indirectly via human-induced simplification of landscape texture.

Most likely, a combination of mechanisms underlies the correlation between landscape texture and avian body mass reported in this study. Regardless of the precise mechanisms, our work strongly supports the relevance of the textural discontinuity hypothesis as a conceptual model to study patterns in avian body mass at the landscape scale.

\section{THE ROLE OF LANDSCAPE TEXTURE IN CONSERVATION BIOGEOGRAPHY}

An increasing amount of evidence suggests that landscape texture is correlated with the distribution of animals (Gunnarson, 1992; Holling, 1992; Telleria \& Carrascal, 1994; McAbendroth et al., 2005; this paper). The notion of landscape texture therefore promises to play a useful role in the development of conservation biogeography. Further empirical investigations of the textural discontinuity hypothesis may contribute to a more refined understanding of the processes driving the distributional patterns of animals - which is a key challenge for conservation biogeography (Whittaker et al., 2005).

At an applied level, the management of landscape texture may be a powerful but largely overlooked tool to influence species composition in a way that is consistent with the conservation priorities for a given landscape. For example, the planting of shrubs in Australian agricultural landscapes can help to attract small birds of conservation concern, while at the same time discouraging colonization by larger and potentially undesirable species, such as the aggressive noisy miner (Manorina melanocephala) (Hastings \& Beattie, 2006). In contrast, animal species of conservation concern that are characteristic of open savannas in southern Africa are threatened by the encroachment of shrubs (Meik et al., 2002; Borghesio \& Giannetti, 2005). The textural discontinuity hypothesis offers a possible framework that may help to assess which species are most likely to gain or lose from changes to landscape management.

The textural discontinuity hypothesis is a pattern-orientated framework, and will be most useful when applied in combination with other landscape concepts such as the notion of landscape mosaics or the Continua-Umwelt concept (e.g. Manning et al., 2004; Tscharntke et al., 2005; Bennett et al., 2006). The textural discontinuity hypothesis is most likely to lead to general insights about communities and assemblages, but is likely to have limited predictive power for individual species (such as the mistletoebird in our data sets). Notwithstanding this caveat, and other things being equal, we formulate the following testable propositions to stimulate future work:

Proposition 1: Collectively, the conservation of small species may be promoted by enhancing the complexity of landscape texture, whereas the conservation of large species can be promoted by open, simple landscape texture. 
Proposition 2: Landscape heterogeneity may benefit species diversity because it provides for a range of conditions suitable to a mix of small and large species.

Proposition 3: Land-use intensification may be a major threat to small species because it homogenizes landscape texture.

Assessing these propositions may strengthen the theoretical foundation of new approaches to conservation in humanmodified landscapes that go beyond the binary classification of land into suitable vs. unsuitable areas.

\section{ACKNOWLEDGEMENTS}

We are grateful to the Canberra Ornithologists Group as well as Damian Michael, Chris Macgregor and Mason Crane for their assistance with the bird surveys. John Stein assisted with preparing the map of the study area. We greatly appreciate the constructive suggestions by two anonymous referees.

\section{REFERENCES}

Allen, C.R. (2006) Discontinuities in ecological data. Proceedings of the National Academy of Sciences USA, 103, 6083-6084.

Allen, C.R., Forys, E.A. \& Holling, C.S. (1999) Body mass patterns predict invasions and extinctions in transforming landscapes. Ecosystems, 2, 114-121.

Allen, C.R., Garmestani, A.S., Havlicek, T.D., Marquet, P.A., Peterson, G.D., Restrepo, C., Stow, C.A. \& Weeks, B.E. (2006) Patterns in body mass distributions: sifting among alternative hypotheses. Ecology Letters, 9, 630-643.

August, P.V. (1983) The role of habitat complexity and heterogeneity in structuring tropical mammal communities. Ecology, 64, 1495-1507.

Bennett, A.F., Radford, J.Q. \& Haslem, A. (2006) Properties of land mosaics: implications for nature conservation in agricultural environments. Biological Conservation, 133, 250264.

Borghesio, L. \& Giannetti, F. (2005) Habitat degradation threatens the survival of the Ethiopian bush crow Zavattariornis stresemanni. Oryx, 39, 44-49.

Brown, J.H., Marquet, P.A. \& Taper, M.L. (1993) Evolution of body size: consequences of an energetic definition of fitness. The American Naturalist, 142, 573-584.

Cardillo, M. \& Bromham, L. (2001) Body size and risk of extinction in Australian mammals. Conservation Biology, 15, 1435-1440.

Cardillo, M., Mace, G.M., Jones, K.E., Bielby, J., BinindaEmonds, O.R.P., Sechrest, W., Orme, C.D.L. \& Purvis, A. (2005) Multiple causes of high extinction risk in large mammal species. Science, 309, 1239-1241.

Cunningham, R.B., Lindenmayer, D.B., Nix, H.A. \& Lindenmayer, B.D. (1999) Quantifying observer heterogeneity in bird counts. Australian Journal of Ecology, 24, 270-277.

Daily, G.C. (2001) Ecological forecasts. Nature, 411, 245.

Davies, J.N., Marchant, S. \& Higgins, P.J. (eds) (1991) Handbook of Australian, New Zealand and Antarctic birds. Volume 1: Ratites to ducks. Oxford University Press, Melbourne.
De la Montana, E., Rey-Benayas, J.M. \& Carrascal, L.M. (2006) Response of bird communities to silvicultural thinning of Mediterranean maquis. Journal of Applied Ecology, 43, 651659.

Diamond, J.M. (1975a) The island dilemma: lessons of modern biogeographic studies for the design of natural reserves. Biological Conservation, 7, 129-145.

Diamond, J.M. (1975b) Assembly of species communities. Ecology and evolution of communities (ed. by M.L. Cody and J.M. Diamond), pp. 342-444. Harvard University Press, London.

Fischer, J. \& Lindenmayer, D.B. (2002) The conservation value of paddock trees for birds in a variegated landscape in southern New South Wales. 2. Paddock trees as stepping stones. Biodiversity and Conservation, 11, 833-849.

Ford, H.A. (1985) A synthesis of the foraging ecology and behaviour of birds in eucalypt forests and woodlands. Birds of eucalypt forest and woodlands: ecology, conservation and management (ed. by A. Keast, H.F. Recher, H. Ford and D. Saunders), pp. 249-254. Royal Australasian Ornithologists Union and Surrey Beatty and Sons, Chipping Norton, New South Wales, Australia.

Gardezi, T. \& da Silva, J. (1999) Diversity in relation to body size in mammals: a comparative study. The American Naturalist, $153,110-123$.

Grover, D.R. \& Slater, P.J. (1994) Conservation value to birds of remnants of Melaleuca forest in suburban Brisbane. Wildife Research, 21, 433-444.

Gunnarsson, B. (1992) Fractal dimension of plants and body size distribution in spiders. Functional Ecology, 6, 636-641.

Haila, Y. (2002) A conceptual genealogy of fragmentation research: From island biogeography to landscape ecology. Ecological Applications, 12, 321-334.

Hastings, R.A. \& Beattie, A.J. (2006) Stop the bullying in the corridors: Can including shrubs make your revegetation more Noisy Miner free? Ecological Management and Restoration, 7, 105-112.

Higgins, P.J. (ed) (1999) Handbook of Australian, New Zealand and Antarctic birds. Volume 4: Parrots to dollarbird. Oxford University Press, Melbourne.

Higgins, P.J. \& Davies, J.N. (eds) (1996) Handbook of Australian, New Zealand and Antarctic birds. Volume 3: Snipe to pigeons. Oxford University Press, Melbourne.

Higgins, P.J. \& Peter, J.M. (eds) (2003) Handbook of Australian, New Zealand and Antarctic birds. Volume 6: Pardalotes to shrike-thrushes. Oxford University Press, Melbourne.

Higgins, P.J., Peter, J.M. \& Cowling, S.J. (eds) (2006) Handbook of Australian, New Zealand and Antarctic birds. Volume 7: Boatbill to starlings. Oxford University Press, Melbourne.

Higgins, P.J., Peter, J.M. \& Steele, W.K. (eds) (2001) Handbook of Australian, New Zealand and Antarctic birds. Volume 5: Tyrantflycatchers to chats. Oxford University Press, Melbourne.

Holling, C.S. (1992) Cross-scale morphology, geometry, and dynamics of ecosystems. Ecological Monographs, 62, 447-502.

Hubbell, S.P. (1997) A unified theory of biogeography and relative species abundance and its application to tropical rain forests and coral reefs. Coral Reefs, 16, S9-S21. 
Hutchinson, G.E. \& Macarthur, R.H. (1959) A theoretical ecological model of size distributions among species of animals. The American Naturalist, 93, 117-125.

Hylander, K., Nilsson, C., Jonsson, B.G. \& Gothner, T. (2005) Differences in habitat quality explain nestedness in a land snail meta-community. Oikos, 108, 351-361.

Kupfer, J.A., Malanson, G.P. \& Franklin, S.B. (2006) Not seeing the ocean for the islands: the mediating influence of matrixbased processes on forest fragmentation effects. Global Ecology and Biogeography, 15, 8-20.

Lindenmayer, D.B., Cunningham, R.B., Donnelly, C.F., Nix, H.A. \& Lindenmayer, B.D. (2002) Effects of forest fragmentation on bird assemblages in a novel landscape context. Ecological Monographs, 72, 1-18.

Lindenmayer, D.B., Cunningham, R.B., MacGregor, C., Tribolet, C. \& Donnelly, C.F. (2001) A prospective longitudinal study of landscape matrix effects on fauna in woodland remnants. I. Experimental design and baseline data. Biological Conservation, 101, 157-169.

Lindenmayer, D.B., Cunningham, R.B. \& McCarthy, M.A. (1999) The conservation of arboreal marsupials in the montane ash forests of the central highlands of Victoria, south-eastern Australia. VIII. Landscape analysis of the occurrence of arboreal marsupials. Biological Conservation, 89, 83-92.

Lindenmayer, D.B. \& Franklin, J. (2002) Conserving forest biodiversity. Island Press, Covelo, California.

MacArthur, R.H. \& Wilson, E.O. (1967) The theory of island biogeography. Princeton University Press, Princeton, New Jersey.

Manning, A.D., Lindenmayer, D.B. \& Nix, H.A. (2004) Continua and Umwelt: novel perspectives on viewing landscapes. Oikos, 104, 621-628.

Marchant, S., Higgins, P.J. \& Davies, J.N. (eds) (1994) Handbook of Australian, New Zealand and Antarctic birds. Volume 2: Raptors to lapwings. Oxford University Press, Melbourne.

McAbendroth, L., Ramsay, P.M., Foggo, A., Rundle, S.D. \& Bilton, D.T. (2005) Does macrophyte fractal complexity drive invertebrate diversity, biomass and body size distributions? Oikos, 111, 279-290.

McIntyre, S. \& Hobbs, R. (1999) A framework for conceptualizing human effects on landscapes and its relevance to management and research models. Conservation Biology, 13, 1282-1292.

Meik, J.M., Jeo, R.M., Mendelson, J.R. \& Jenks, K.E. (2002) Effects of bush encroachment on an assemblage of diurnal lizard species in central Namibia. Biological Conservation, 106, 29-36.

Meir, E., Andelman, S. \& Possingham, H.P. (2004) Does conservation planning matter in a dynamic and uncertain world? Ecology Letters, 7, 615-622.

Miles, D.B. \& Ricklefs, R.E. (1984) The correlation between ecology and morphology in deciduous forest passerine birds. Ecology, 65, 1629-1640.

Patterson, B.D. (1987) The principle of nested subsets and its implications for biological conservation. Conservation Biology, 1, 323-334.

Polo, V. \& Carrascal, L.M. (1999) Shaping the body mass distribution of Passeriformes: habitat use and body mass are evolutionarily and ecologically related. Journal of Animal Ecology, 68, 324-337.

Prober, S.M. \& Thiele, K.R. (1995) Conservation of the Grassy White Box Woodlands: relative contributions of size and disturbance to floristic composition and diversity of remnants. Australian Journal of Botany, 43, 349-366.

Renjifo, L.M. (1999) Composition changes in a subandean avifauna after long-term forest fragmentation. Conservation Biology, 13, 1124-1139.

Reynolds, J.D. (2003) Life histories and extinction risk. Macroecology: concepts and consequences (ed. by T.M. Blackburn and K.J. Gaston), pp. 195-217. Blackwell Publishing, Oxford.

Ricketts, T.H. (2001) The matrix matters: effective isolation in fragmented landscapes. The American Naturalist, 158, 8799.

Roland, J. \& Taylor, P.D. (1997) Insect parasitoid species respond to forest structure at different spatial scales. Nature, 386, 710713.

Schoener, T.W. (1968) Sizes of feeding territories among birds. Ecology, 49, 123-141.

Siemann, E. \& Brown, J.H. (1999) Gaps in mammalian body size distributions reexamined. Ecology, 80, 2788-2792.

Telleria, J.L. \& Carrascal, L.M. (1994) Weight-density relationships between and within bird communities - implications of niche space and vegetation structure. The American Naturalist, 143, 1083-1092.

Tscharntke, T., Klein, A.M., Kruess, A., Steffan-Dewenter, I. \& Thies, C. (2005) Landscape perspectives on agricultural intensification and biodiversity - ecosystem service management. Ecology Letters, 8, 857-874.

Watson, J.E.M., Whittaker, R.J. \& Freudenberger, D. (2005) Bird community responses to habitat fragmentation: how consistent are they across landscapes? Journal of Biogeography, 32, 13531370.

Whittaker, R.J., Araujo, M.B., Paul, J., Ladle, R.J., Watson, J.E.M. \& Willis, K.J. (2005) Conservation biogeography: assessment and prospect. Diversity and Distributions, 11, 3-23.

Wiens, J.A. (1989) Spatial scaling in ecology. Functional Ecology, 3, 385-397.

\section{SUPPLEMENTARY MATERIAL}

The following supplementary material is available for this article:

Table S1 The body mass values and numbers of detections for all bird species.

This material is available as part of the online article from: http://www.blackwell-synergy.com/doi/abs/10.1111/ j.1472-4642.2007.00411.x

(This link will take you to the article abstract).

Please note: Blackwell Publishing is not responsible for the content or functionality of any supplementary materials supplied by the authors. Any queries (other than missing material) should be directed to the corresponding author for the article. 